\title{
ON THE COVARIANCE STRUCTURE AND SIMULTANEOUS COMPARISONS OF THE ESTIMATES OF AHP-WEIGHTS
}

\author{
Pertti Laininen \\ Helsinki University of Technology, Systems Analysis Laboratory, P.O.Box 1100, Fin-02015 Hut, \\ Finland, Telefax: +358-9-4513096 \\ E-mail: Pertti.Laininen@ hut.fi
}

\begin{abstract}
We discuss the estimation of local weights $w_{1}, \ldots, w_{m}$ of $m$ attributes or decision alternatives from a $m \times m$ AHP-matrix including the pairwise comparison ratios $r_{i j},\left(r_{j i}=1 / r_{i j}\right), i, j=1, \ldots, m$. The ratios $r_{i j}$ given by the decision maker are done by using integers $1, \ldots, 9$. The values of the ratios are values of random variables, and so are the estimates $\hat{w}_{1}, \ldots, \hat{w}_{m}$. The joint distribution of the estimates is very complicated, and it is has not been completely treated in the literature. One approach using the eigenvector method can be found in Vargas (1982). We characterize the covariance (correlation) structure of the estimates by introducing the following model
\end{abstract}

$$
\log \left(r_{i j}\right) \sim \mathrm{N}\left(\log \left(v_{i}\right)-\log \left(v_{j}\right), \sigma^{2}\right) .
$$

Here $\sigma^{2}$ is a constant variance, and $v_{i}$ is the value of attribute $i$. There are experiences where the normal distribution of $\log \left(r_{i j}\right)$ is evident. A good collection of the actual AHP-matrices is given by Leskinen (2000). The connection between the weights and the values is $w_{i}=v_{i} /\left(v_{1}+\ldots+v_{m}\right)$, $i=1, \ldots, m$. Writing $\log \left(v_{i}\right)=\beta_{i}$ one obtains

$$
w_{i}=\frac{e^{\beta_{i}}}{e^{\beta_{1}}+\ldots+e^{\beta_{m}}}, \quad i=1, \ldots, m
$$

By using a regression analysis the estimates $\hat{\beta}_{i}, i=1, \ldots, m$, and the covariances $\operatorname{Cov}\left(\hat{\beta}_{i}, \hat{\beta}_{j}\right), i, j=1, \ldots, m$ can be easily calculated. We solve the regression problem with the constraint $\beta_{m}=0$. From these results the estimates $\hat{w}_{i}=e^{\hat{\beta}_{i}} /\left(e^{\hat{\beta}_{1}}+\ldots+e^{\hat{\beta}_{m}}\right), i=1, \ldots, m$, the covariances $\operatorname{Cov}\left(\hat{w}_{i}, \hat{w}_{j}\right), i, j=1 .,,, m$ can be evaluated.

Because the variables $e^{\hat{\beta}_{i}}, i=1, \ldots, m-1$ follow lognormal distribution one can derive

$$
\operatorname{Cov}\left(e^{\hat{\beta}_{i}}, e^{\hat{\beta}_{j}}\right)=e^{\beta_{i}+\operatorname{Cov}\left(\hat{\beta}_{i}, \hat{\beta}_{i}\right) / 2} e^{\beta_{j}+\operatorname{Cov}\left(\hat{\beta}_{j}, \hat{\beta}_{j}\right) / 2}\left(e^{\operatorname{Cov}\left(\hat{\beta}_{i}, \hat{\beta}_{j}\right)}-1\right)
$$

Through linearization of the formula of $w_{i}$ with respect to $e^{\beta_{j}}$ one obtains

$$
\operatorname{COV}\left(\hat{w}_{i}, \hat{w}_{j}\right)=\left[\frac{\partial w_{i}}{\partial e^{\beta_{j}}}\right]_{m \times(m-1)} \operatorname{COV}\left(e^{\hat{\beta}_{i}}, e^{\hat{\beta}_{j}}\right)_{(m-1) \times(m-1)}\left[\frac{\partial w_{i}}{\partial e^{\beta_{j}}}\right]^{T}{ }_{(m-1) \times m}
$$


where

$$
\left[\frac{\partial w_{i}}{\partial e^{\beta_{j}}}\right]_{m \times(m-1)}=-w_{m}\left[\begin{array}{cccc}
w_{1}-1 & w_{1} & \cdots & w_{1} \\
w_{2} & w_{2}-1 & \cdots & w_{2} \\
\cdots & \cdots & \cdots & \cdots \\
w_{m-1} & w_{m-1} & \cdots & w_{m-1}-1 \\
w_{m} & w_{m} & \cdots & w_{m}
\end{array}\right]
$$

The estimate $\hat{\operatorname{Cov}}\left(\hat{w}_{i}, \hat{w}_{j}\right)$ is calculated by putting the estimates $\hat{\operatorname{Cov}}\left(e^{\hat{\beta}_{i}}, e^{\hat{\beta}_{j}}\right)$ and $\hat{w}_{i}, i, j=1, \ldots, m$ into the matrices above.

A special feature of the covariance matrix $\operatorname{COV}\left(\hat{w}_{i}, \hat{w}_{j}\right)$ is that the covariances are dependent on the weights $w_{1}, \ldots, w_{m}$. This means for instance that if one wants to test a hypothesis $H_{0}: w_{i}=w_{j}$ the test statistic $\left(\hat{w}_{i}-\hat{w}_{j}\right) / \operatorname{Se}\left(\hat{w}_{i}-\hat{w}_{j}\right)$ is dependent on the values of $w_{1}, \ldots, w_{m}$ under $H_{0}$. Therefore, it is easier to test $H_{0}: \beta_{i}=\beta_{j}$ by using the test statistic $\left(\hat{\beta}_{i}-\hat{\beta}_{j}\right) / \operatorname{Se}\left(\hat{\beta}_{i}-\hat{\beta}_{j}\right)$ which is not dependent on the values of $\beta_{1}, \ldots, \beta_{m}$ under $H_{0}$.

We present here how to make simultaneous comparisons of the weights $w_{1}, \ldots, w_{m}$. The test of the hypothesis $H_{0}: \beta_{1}=\ldots=\beta_{m}$ (i.e. $H_{0}: w_{1}=\ldots=w_{m}$ ) at the risk level 0.05 tells how well the decision maker is able to separate different alternatives under comparisons. $H_{0}$ can be tested by checking simultaneously all hypothesis $H_{0}: \beta_{i}=\beta_{j}, i \neq j=1, \ldots, m$ by using the test statistic

$$
t_{i j}=\frac{\hat{\beta}_{i}-\hat{\beta}_{j}}{\operatorname{Se}\left(\hat{\beta}_{i}-\hat{\beta}_{j}\right)}
$$

that follows the t-distribution with $n-m+1$ degrees of freedom. In simultaneous testing the critical value $t_{m}(0.05)$ for $t_{i j}$ is the value that $\max \left|t_{i j}\right|$ exceeds with probability 0.05 . Critical values calculated by simulations are given in the following table.

$\begin{array}{lllllllll}m & 3 & 4 & 5 & 6 & 7 & 8 & 9 & 10 \\ t_{m}(0.05) & 18.94 & 4.79 & 3.76 & 3.46 & 3.39 & 3.34 & 3.36 & 3.37\end{array}$

\section{References}

Leskinen, P., 2000. Measurement Scales and Scale Independence in the Analytic Hierarchy Process. J. Multi-Crit. Decis. Anal. 9: 163-1674.

Vargas, L.G., 1982. Reciprocal Matrices with Random Coefficients. Mathematical Mode. 3: 69-81. 